\title{
Research on Intergenerational Knowledge Transfer under the Chinese Context Multinationals' Leaders
}

\author{
Xiaohui Jing \\ School of Management, Wuhan University of Technology, Wuhan 430070, China \\ 1242908769@qq.com
}

\begin{abstract}
With the development of economic globalization and Chinese economy, MNCs, as main participants of global direct investment, China has been the core organizer of international economic activities. Along with the rising of knowledge-based economy, the knowledge management gradually became the theme of enterprise management. As an important part of knowledge management process, the knowledge transfer becomes the essential foundation of many organizations to construct the competitive advantage. This thesis is a case study on the individual to the organization's knowledge transfer, by identifying the factors that influences the MNCs CEO's knowledge transfer performance, makes a research on the case of enterprises and builds influence factor model, finally puts forward policy suggestions for training and incentive for MNC.
\end{abstract}

Keywords: Multinational Corporations (MNC); Chinese Situation; Intergenerational Succession; Knowledge Transfer.

\section{中国情境下跨国公司领导者的代际知识转移一基于佛吉亚（武汉）的案例研究}

\author{
敬晓慧 \\ 武汉理工大学管理学院, 武汉 中国
}

摘 要: 随着经济全球化和中国经济的崛起, 跨国公司在中国投资的热潮不断升温, 出现大量 在中国设子公司的跨国公司, 伴随知识更新速度的加快, 知识管理逐渐成为企业管理的核心 内容, 知识转移作为知识管理的关键环节, 已成为跨国公司打造竞争优势的关键所在。本文 通过个体对组织知识转移绩效影响的探索性案例研究, 识别影响跨国公司领导者代际知识转 移绩效的要素, 构建影响因素模型, 并对跨国公司继任领导者提出培养和激励的政策性建议。

关键词：跨国公司；中国情境；代际；知识转移

\section{1. 前言}

知识转移是个体之间的一项学习活动, 学习的知识存在于不同的层次, 并非每个公司成员 都乐于且善于将个人拥有的知识与其它成员分享，况且成功的最佳实践也不是能够简单清楚 的表达和传递，尤其是公司核心领导人物代际之间的知识能否实现顺畅转移至关重要 [1]。有 众多学者对跨国公司内部知识转移进行研究, 但对跨国公司中个体层面的知识转移方面的研 究还非常少。本文围绕 “如何实现中国情境下跨国公司领导者的代际知识顺畅转移” ，基于 佛吉亚公司进行探索性案例分析, 识别个体之间知识转移的影响因素, 构建出影响因素修正 模型, 并通过文献研究和案例研究以及理论研究的方式, 提出了如何实现跨国公司领导者代 际知识的顺畅转移，有益于促进人力资源管理理论的实践和发展。

\section{2. 影响因素分析及模型构建}

本文从知识转移的主体、知识和组织三个角度来研究中国情境下跨国公司领导者的代际 知识转移, 对影响知识转移绩效的因素进行归纳整理。从主体层面, 即现任领导者角度而言, 对与继任领导者之间知识转移的意愿的程度，作为领导者他的领导风格以及他应对知识转移 
的能力强弱都会直接影响到他与继任领导者之间的知识转移 [2]。知识转移的接收方即为继任 领导者的接收意愿和接收能力也将直接影响代际知识的接收绩效。

许多研究学者普遍认为知识本身的特质是造成知识转移困难的影响因素之-, 尤其是隐性 知识不易表达、高度个人化, 依赖-定的社会情境形成等特征。本文从知识缄默性、复杂性、 专用性、可靠性、知识距离这几方面具体分析是如何对代际知识转移造成影响的 [3]。

从知识转移的的情境来讲, 本文把知识转移的主体所在的组织作为知识发生转移的情境, 学习型组织和关系友好型组织是跨国公司领导者代际知识转移的最理想的发生情境 [4]。在学 习型组织中, 所有的员工都是处在积极学习的氛围中, 对于知识的发送和接收都是报以主动 的态度, 这也极大促进了公司代际间知识的转移。关系友好型组织中营造的友好型员工关系, 在这样的情境中知识发生转移的阻碍会大大减少, 进而产生便利的渠道来进行知识转移。知 识的距离是指知识转移双方之间知识存量的差距, 知识存量差距越小, 代际之间的知识转移 的效果就越理想, 反之就会对知识转移效果产生-定的阻碍 [5]。

本文在归纳国内外关于代际知识转移相关文献以及分析上述影响跨国公司代际传承中知识转 移的因素的基础上，构建了相应的影响因素模型。如图 1。

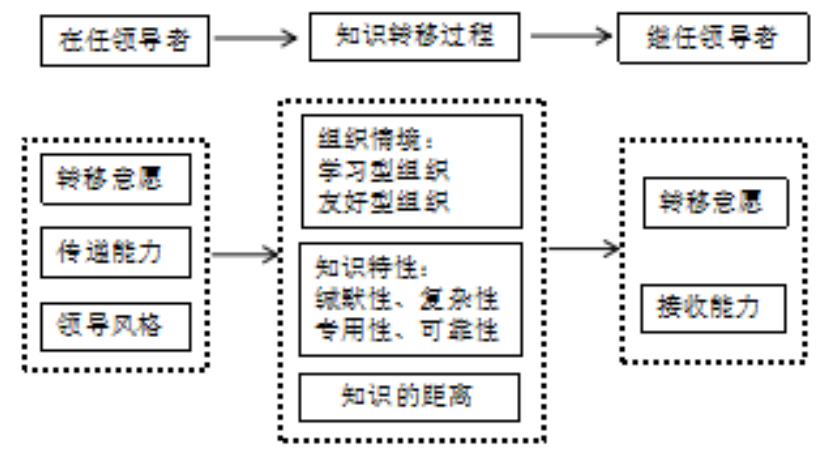

图1 中国情境下跨国公司领导者代际知识转移的影响因素模型

\section{3. 探索性案例研究}

\section{1 案例选择}

与实验、问卷调查、文档分析等并列为社会科学领域重要研究方法的案例研究方法 (Case Study Research) 受到国内外众多学者的重视和应用, 建立在对管理现象的来龙去脉进行广泛 调研的基础上, 引导研究者专注于那些没有明确答案, 但非常重要的问题 [6]。本文采用案例 实证, 选择案例时共考虑 3 个因素: (1)限定为跨国公司: (2)该跨国公司在中国有分公司, 得 到行业内的公认, 享有较高声誉; (3)信息的可获得性。在这 3 个标准的基础上, 最终选择了 佛吉亚武汉分公司作为本文的案例研究公司。

法国佛吉亚集团公司（持股 51\%）及台湾全兴集团公司（持股 49\%）合资经营的佛吉亚全 兴 (武汉) 座椅有限公司始建于 2002 年 8 月, 至今已经历了 7 代领导人的更替, 大概每代领 导人的在职时间为 2 年, 每-任新的领导者通常是由上级根据绩效和能力直接任命的, 而公司 在任的原领导者则被提拔到中国区域更高的层级。在公司领导人的卓越领导下, 佛吉亚全兴

（武汉）座椅有限公司被神龙汽车颁发 “总裁特别奖” , 对佛吉亚（武汉）公司领导人给予 重要肯定。

3.2 数据搜集

在案例研究中常采用多种方法进行数据搜集, 除-般量化研究方法之外, 还包含各种质化 方法。本次案例研究采用了深度访谈、信息查询、文件调阅、直接观察及媒体资料等方法收 集数据。本研究中获得的主要信息是通过访谈, 在信息采集过程中, 对佛吉亚全兴（武汉） 座椅有限公司的现任 CEO 进行了半结构化的深度访谈。本研究中的另一个信息根源是文件调 阅。在被访谈者提供的公司内部资料, 如内部刊物、工作总结和汇报材料中采集大量数据。 充分借助在佛吉亚全兴（武汉）公司实习的机会, 尤其是本届新任领导者上任之际, 我对本 
案例进行了较为深入的跟踪研究, 通过现场考察、参加中高层管理人员报告会等方式获得了 大量较为客观的-手资料。此外通过公司网站、公司年报、宣传手册和业界新闻等公开的信息 源收集了公司大量的一手资料。

3.3 数据分析

在案例研究中, 数据资料分析是最为重要及复杂的部分, 采用内容分析法对本次研究中 的案例数据依次进行编码。根据 Glaser 和 Strauss（1998）的观点, 数据的搜集与分析是相 辅相成的, 而非彼此之间相互独立。本次研究内容主要包括：对案例公司的领导人代际知识 转移主体、知识特性、组织文化和知识的转移程度等主要研究构念, 同时参照各构念的子类 别对数据做进-步的编码, 最后根据案例编码对案例公司代际知识转移影响因素模型进行修 正，并分析归纳出适合子公司在中国的跨国公司领导者如何顺畅实现代际知识转移。

3.4 案例编码

本研究参照郑伯损（2005）建议的步骤，根据访谈、文件等资料建立文本，结合数据分 析的结果, 将所有数据进行归类, 发展编码类别, 对案例企业的主体层面、知识层面、组织 层面进行案例编码。

表1 案例企业的主体层面编码结果

\begin{tabular}{|c|c|}
\hline 主体层面 & 编码例证 \\
\hline 知识发送者 & 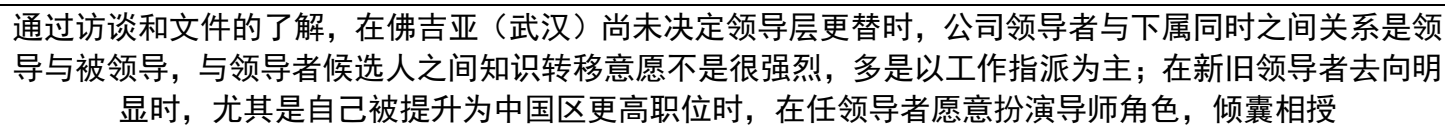 \\
\hline 知识接收者 & 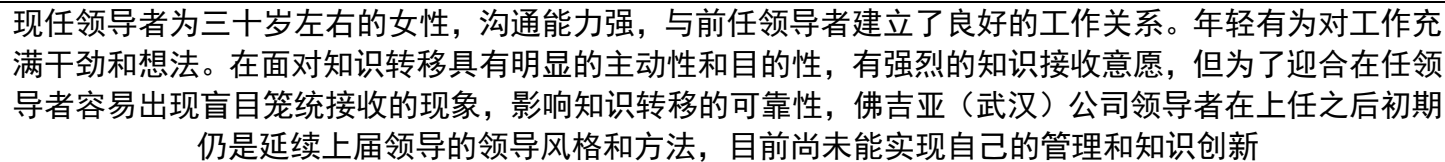 \\
\hline
\end{tabular}

表2 案例企业的知识层面编码结果

\begin{tabular}{|c|c|}
\hline 知识层面 & 编码例证 \\
\hline 知识特性 & 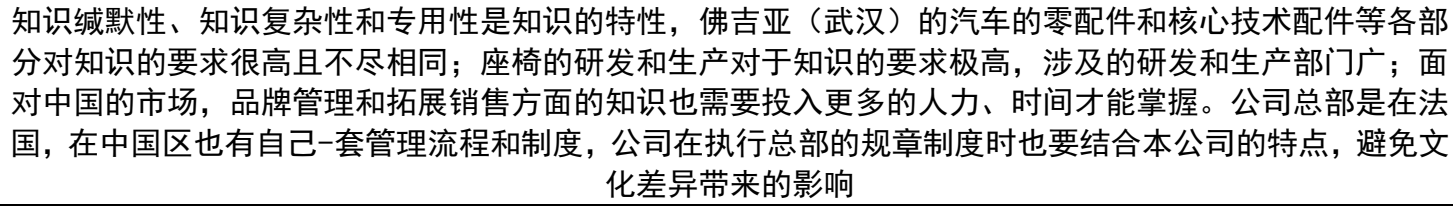 \\
\hline 知识距离 & $\begin{array}{c}\text { 在任领导者工作经验及其丰富, 虽然学历没有继任领导者高, 但针对佛吉亚（武汉）的专业知识存量高于 } \\
\text { 在任领导者, 从访谈中得知, 双方都是在中国区佛吉亚工作5年以上的, 也都从事管理工作, 双方之间并不 } \\
\text { 存在过大的知识差距 }\end{array}$ \\
\hline $\begin{array}{l}\text { 知识的 } \\
\text { 可靠性 }\end{array}$ & $\begin{array}{c}\text { 佛吉亚（武汉）公司有健全的企业文化, 极强的凝聚力, 在公司领导人的卓越领导下, } 2011 \text { 年佛吉亚全兴 } \\
\text { (武汉) 座椅有限公司被它的主要客户神龙汽车颁发 “总裁特别奖”, 足以证明领导者之间转移的知识是 } \\
\text { 足以信赖的, 被证实为有见识的, 能给公司带来-定收益的 }\end{array}$ \\
\hline
\end{tabular}

表3 案例企业的组织层面编码结果

\begin{tabular}{|c|l|}
\hline 组织层面 & \multicolumn{1}{|c|}{ 编码例证 } \\
\hline & $\begin{array}{l}\text { 公司坚持贯彻团队合作、透明度、企业家精神、持续改进、尽职尽责五位-体的价值观。在人文建设方面, } \\
\text { 逐渐形成了 “五没有” 精神: 没有借口, 没有理由, 没有粉饰, 没有内使, 没有例外。公司实行员工个人 } \\
\text { 合理化建议, 并积极鼓励创新; 公司有自己内部的期刊杂志; 公司领导和员工同-餐厅用餐, 形成良好的员 } \\
\text { 工关系, 员工生日公司领导者会亲自给予祝福并送出贴心礼物, 实现员工 “快乐生活 快乐工作” 的愿望 }\end{array}$ \\
\hline 组织氛围 & $\begin{array}{l}\text { 在佛吉亚 (武汉) 实习的日子里, 我切身感受到公司的学习氛围和友好关系, 公司为每位新进员工发放员 } \\
\text { 工手册, 公司会定期组织免费专家培训, 也会举行 “家庭友好日” 活动。在这样的组织氛围下以及非正式 } \\
\text { 交流活动中, 是非常容易实现彼此间的知识转移的 }\end{array}$ \\
\hline
\end{tabular}

3.5 影响因素修正模型

从知识转移的主体层面、知识层面和组织层面对案例公司进行编码分析，结合影响跨国 公司领导者代际知识转移绩效的因素分析，对影响因素模型加以补充修正，影响因素修正模 型如图 2。 


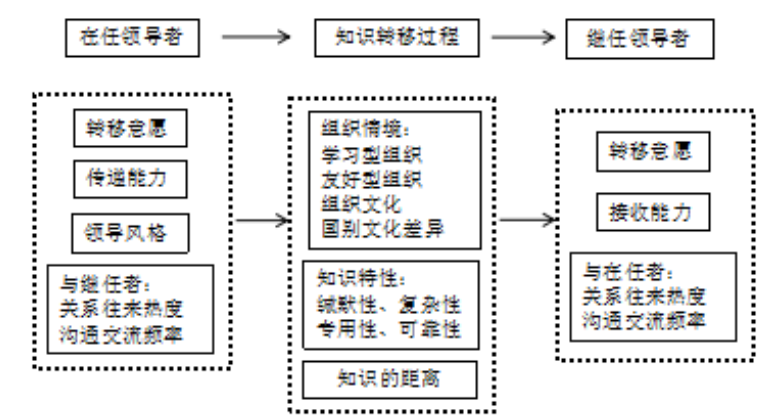

图2 中国情景下跨国公司领导者间代际知识转移的影响因素修正模型

从案例分析得出, 知识转移的主体层面中在任领导者和继任领导者之间的关系往来热度 和沟通交流频率, 也是影响双方之间代际知识转移的因素 [7]。在中国情境下, 人情和关系这 样的网络依然作用于中国情境下的跨国公司, 关系往来热度主要是指双方的非正式非群体交 往, 在任领导者与继任领导者的交往越密切, 双方之间的知识转移发生的频率就越高, 转移 的场所可以是工作场所, 也可以是生活场所。沟通交流频率主要是指知识转移的双方关于工 作关于管理工作的交流, 双方关于企业管理的经验交流越多, 继任领导者对于对所吸收的代 际知识运用得就更得心应手。

从组织层面来讲, 组织文化和国别文化差异也作用与跨国公司领导者的代际知识转移绩 效。组织文化作为公司-种无形资产, 它所产生的效益确实及其重大的, 案例企业的 “五个价 值观” 以及 “五没有” 都对公司领导者的代际知识的转移产生了影响。另外，对于跨国公司 来说, 国别文化的差异是不可以忽略的, 尤其是在公司领导层新老交替时, 如果代际知识转 移的双方不是一个来自于一个国家, 那么双方之间的知识转移的困难就会大幅度增加, 因为双 方的生活背景、文化背景、交流方式、思维模式等等都会有明显的差异, 而这些差异就会构 成影响双方代际知识转移绩效的因素 [8]。

\section{4. 促进跨国公司领导者代际知识转移及继承者的培养建议}

4.1 强化公司知识分享的氛围及员工参与的热情

根据计划行为理论, 知识转移中个体知识转移行为受多方面因素的影响, 包括组织中上 级领导和同事对知识转移的支持程度, 以及个体对知识转移过程中完成所承担任务的信心等 [9]。跨国公司通过建立适当的制度, 共享组织文化、激励机制安排, 同时积极推广知识转移 成功的经验, 显著的提升了跨国公司领导者和员工参与知识转移行为的意愿水平, 从而推动 知识转移的有效推进和成功实施。

4.2 积极完善跨国公司领导者的代际知识转移方法

知识管理界关于知识转移方法已形成基本共识，即同时采用基于技术和文化的知识管理 方法, 实现编码化策略和人本化策略的共生。虽然代际知识转移从代际特征上对知识转移方 法提出了新的要求, 但代际知识转移方法的选择不离知识转移方法的根本。导师制和知识库 作为以文化为中心和以技术为中心的方法被广泛采用, 通过案例研究发现跨国公司要适度发 挥知识库方法的辅助作用。

4.3 重视在中国情境下非正式群体活动对知识转移的重要性

参照中国市场的实际情况, 在非正式群体活动中, 公司领导者代际传承中默会知识和关 系网络的转移应受到足够重视。跨国公司领导者通过带领公司继任者参加非正式群体的活动, 加强默会知识和人际关系网的自发转移, 这是一种建立在情感相似、兴趣相近、利益相同基础 上的自发的知识转移, 能减轻和解决组织知识转移的有限理性和机会主义问题, 它既能为知 识来源者提供把知识分享出去的激情, 也为知识接收者提供了感受学习的群体氛围。

4.4 鼓励接班人调整心态, 增强务实精神

公司接班人由领导层更替前的追随者变为公司的决策者, 身份角色转变的同时也要求与 之相应的心态和思维的变化。在公司应当采取适当措施鼓励他们主动自我调整心态, 找准定 
位。“新官上任三把火” 是中国无论是政府还是企业都普遍存在的一个现象, 容易出现较为激 进的做法, 造成冒进过世。所以, 要求公司在培养接任领导者时要树立他们的务实精神。

\section{5. 本文的局限及未来研究}

本文的研究存在一定的局限性，部分的研究采用的而是二手资料，未来的研究将寻求掌握 更多的-手访谈资料。同时, 为了提高本研究结果的信度, 需要进-步调研其他行业, 以验证 本文所构建的模型; 此外, 在案例得到验证的基础上, 未来还可以进行大样本调研和统计分 析，从而在更大的范围研究代际知识转

\section{References}

[1] Argote, Ingram,P. Knowledge transfer: a basis for competitive advantage in firms. Organizational behavior and human decision prosses, 2000, 82(1):150-169.

[2] LiBing Shu, The process of knowledge transfer mechanisms within the team and influencing factors [D]. Doctoral Dissertation of Zhejiang University,2006

[3] Teece, DJ. Capturing Value from Knowledge Assets: The New Economy, Markets for Know-How, and Intangrble Assets. California Management Review

[4] Szulanski, G (1996). Exploring internal stickiness: Impediments to the transfer of best practice within the firm[J], Strategic Management Journal, 17(Winter Special Issue): 27-43

[5] Szulanski, The process of behavior and human decision transfer. a diachronic analysis of Stickiness [J], Organizational 2000,82(1):9-27.

[6] Yin, R. K. Case study research: Design and methods. SAGE Publications Incorporated. 2008.

[7] Hansen, M.T. The search-transfer problem: The role of weak ties in sharing knowledge across organizational subunits. Administrative Science Quarterly

[8] Galbraith, C.S, Transferring core manufacting technology in high technology fines [J], California management review, 1990, 32(4):56-70.

[9] Yu Gao, Research on the mechanism of bidirectional knowledge transfer of parent subsidiary companies in Chinese manufacturing industry [D]. Doctoral Dissertation of Zhejiang University, 2013. 